\title{
GOES-16 ABI navigation assessment
}

Bin Tan, John Dellomo, Robert Wolfe, Alan Reth

Bin Tan, John Dellomo, Robert Wolfe, Alan Reth, "GOES-16 ABI navigation assessment," Proc. SPIE 10764, Earth Observing Systems XXIII, 107640G (7 September 2018); doi: 10.1117/12.2321170

SPIE Event: SPIE Optical Engineering + Applications, 2018, San Diego, California, United States 


\title{
GOES-16 ABI Navigation Assessment
}

\author{
Bin $\operatorname{Tan}^{1,2}$, John Dellomo ${ }^{1,3}$, Robert Wolfe $^{1}$, and Alan Reth ${ }^{1,4}$ \\ 1. Goddard Space Flight Center, Greenbelt, MD \\ 2. Science Systems and Applications, Inc., Lanham, MD \\ 3. Global Science \& Technology, Inc., Greenbelt, MD \\ 4. Chesapeake Aerospace, LLC, Grasonville, MD
}

\begin{abstract}
The US Geostationary Operational Environmental Satellite - R Series (GOES-R) was launched on November 19, 2016 and was designated GOES-16 upon reaching geostationary orbit ten days later. After checkout and calibration, GOES-16 was relocated to its operational location of 75.2 degrees west and officially became GOES East on December 18, 2017. The Advanced Baseline Imager (ABI) is the primary instrument on the GOES-R series for imaging Earth's surface and atmosphere to significantly improve the detection and observation of severe environmental phenomena. A team supporting the GOES-R Flight Project at NASA's Goddard Space Flight Center developed algorithms and software for independent verification of ABI Image Navigation and Registration (INR), which became known as the INR Performance Assessment Tool Set (IPATS). In this paper, we will briefly describe IPATS on top concept level, and then introduce the Landsat chips, chip registration algorithms, and how IPATS measurements are filtered. We present GOES-16 navigation (NAV) errors from flight data from January 2017 to May 2018. The results show a) IPATS characterized INR variations throughout the post-launch test phase; and b) ABI INR has improved over time as post-launch tests were performed and corrections applied. Finally, we will describe how estimated NAV errors have been used to assess and understand satellite attitude anomalies and scale errors etc. This paper shows that IPATS is an effective tool for assessing and improving GOES-16 $\mathrm{ABI}$ INR and is also useful for INR long-term monitoring.
\end{abstract}

Keywords: GOES-R, Image Navigation, ABI, Remote Sensing, Satellite

\section{INTRODUCTION}

The US Geostationary Operational Environmental Satellite - R Series (GOES-R) was launched on November 19, 2016 and was designated GOES-16 upon reaching geostationary orbit above 89.5 degrees west longitude ten days later. After checkout and calibration, GOES-16 was relocated to its operational location of 75.2 degrees west and officially became GOES East on December 18, 2017. The Advanced Baseline Imager (ABI) is the primary instrument on the GOES-16 for imaging Earth's surface and atmosphere to significantly improve the detection and observation of severe environmental phenomena [1][2]. The ABI generates Level 1B (L1B) images (or frames) of three types: Full Disk (FD) which spans the entire viewable hemisphere, Continental United States (CONUS) which covers the United States, and Mesoscale (MESO) which can be tasked to any local region. The FD is a circle of angular diameter 17.4 degrees as measured from the satellite location with center at satellite nadir and circumference at the Earth limb. The CONUS images are rectangular and have an extent of $5000 \mathrm{~km}$ in the East-West (EW) direction and $3000 \mathrm{~km}$ in the North-South (NS) direction. The MESO images are also rectangular and have an extent of $1000 \mathrm{~km} \mathrm{EW} \mathrm{x} 1000 \mathrm{~km}$ NS [3].

Earth location or geolocation accuracy is a key quality indicator of the satellite data. Accurate geolocation ensures the data from different channels of a sensor or the data from different sensors/sources can be applied together to retrieve high level biogeophysical information [4][5]. NOAA's satellite data production system registers different scans of ABI data by geometric correction of each scan into the ABI fixed grid coordinate system, a projection based on the viewing perspective of the idealized location of a satellite in geosynchronous orbit [6]. The geolocation accuracy of the gridded GOES-16 data is evaluated through Image Navigation and Registration (INR) assessments, an important part of the post-launch calibration and validation $(\mathrm{Cal} / \mathrm{Val})$ activities. The assessment results are not only used to verify the geolocation accuracy but also

Earth Observing Systems XXIII, edited by James J. Butler, Xiaoxiong (Jack) Xiong, Xingfa Gu, Proc. of SPIE Vol. 10764, 107640G · C 2018 SPIE · CCC code: 0277-786X/18/\$18 · doi: 10.1117/12.2321170 
provide in-depth analysis to help the engineers to improve the geolocation algorithms, operational parameters and future instrument design.

In this paper, we first introduce the INR Performance Assessment Tool Set (IPATS), developed under the auspices of the NASA's GOES-R Flight Project for independent verification of ABI and Geostationary Lightning Mapper (GLM) INR. Then we present GOES-16 navigation (NAV) assessment results for flight data from January 2017 to May 2018. Finally, we present examples of how the estimated NAV errors have been used to assess and understand subtle, unexpected and spurious events or situations such as satellite attitude anomalies, and scale error.

\section{INR PERFORMANCE ASSESSMENT TOOL SET}

The IPATS was designed and developed to support a broad range of ABI INR performance analyses, in particular the generation of the INR performance metrics bounded by requirements stated in the ABI Performance and Operational Requirements Document (PORD) [8]. IPATS was also designed to support analysis of the navigation quality of background images produced by the Geostationary Lightning Mapper (GLM). There are five types of metrics produced by IPATS:

- $\quad$ NAV error (ABI \& GLM): Difference between location of pixel in data product and true location.

- Channel-to-channel registration (CCR) error (ABI): Relative navigation error of corresponding pixels of different channels in the same frame.

- Frame-to-frame registration (FFR) error (ABI): Relative navigation error of corresponding pixels of same channel in consecutive images.

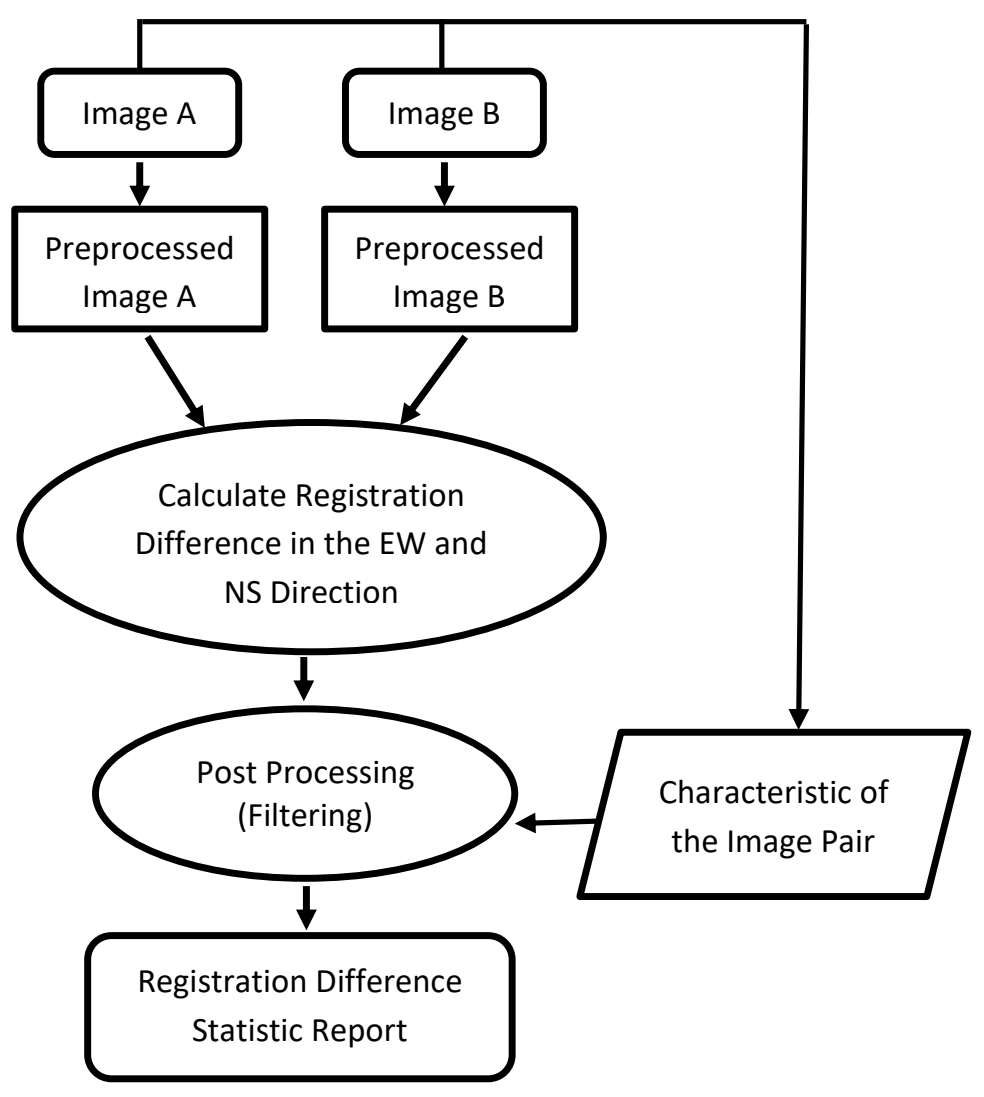

Figure 1. High level algorithm diagram of IPATS. 
- Within-frame registration (WIFR) error (ABI): Difference between radial separation of two pixels on the fixed grid coordinate system and their true angular separation. WIFR is calculated indirectly from the NAV results.

- Swath-to-swath registration (SSR) error (ABI): Relative navigation error of two neighboring pixels on opposite sides of the horizontal image swath boundary.

Figure 1 shows a high level diagram of IPATS. All above metrics, except for WIFR, go through similar modules with different image types, configuration parameters and filters for each metric. Metrics are generated separately for each of the imaging modes: FD, CONUS and MESO. WIFR is an additional step that operates on the NAV results. IPATS uses a modular algorithm architecture with each module containing several user-selectable algorithms. For example, Pearson Cross Correlation, Normalized Mutual Information and Fourier Space Correction algorithms are in the image matching module. Each module is independent and interchangeable and accomplishes the designed functionality with a user-selected algorithm. In the last module, the IPATS raw measurements are filtered to identify high quality measurements that are used to produce the assessment reports. This remainder of paper is focused only on the NAV FD metric.

\subsection{Landsat chips}

The geolocation accuracy of the Landsat images are within 15 meters [9], which is 3\% or less of the spatial resolutions of GOES-16 ABI (Table 1). Therefore, in the ABI NAV measurements, the subsets of Landsat 7 and 8 images, called chips here, are considered the true location. The 630 chips for assessing ABI NAV are mostly along the shoreline of North and South America (Figure 2). More chips will be added as more eligible Landsat 8 images become available.

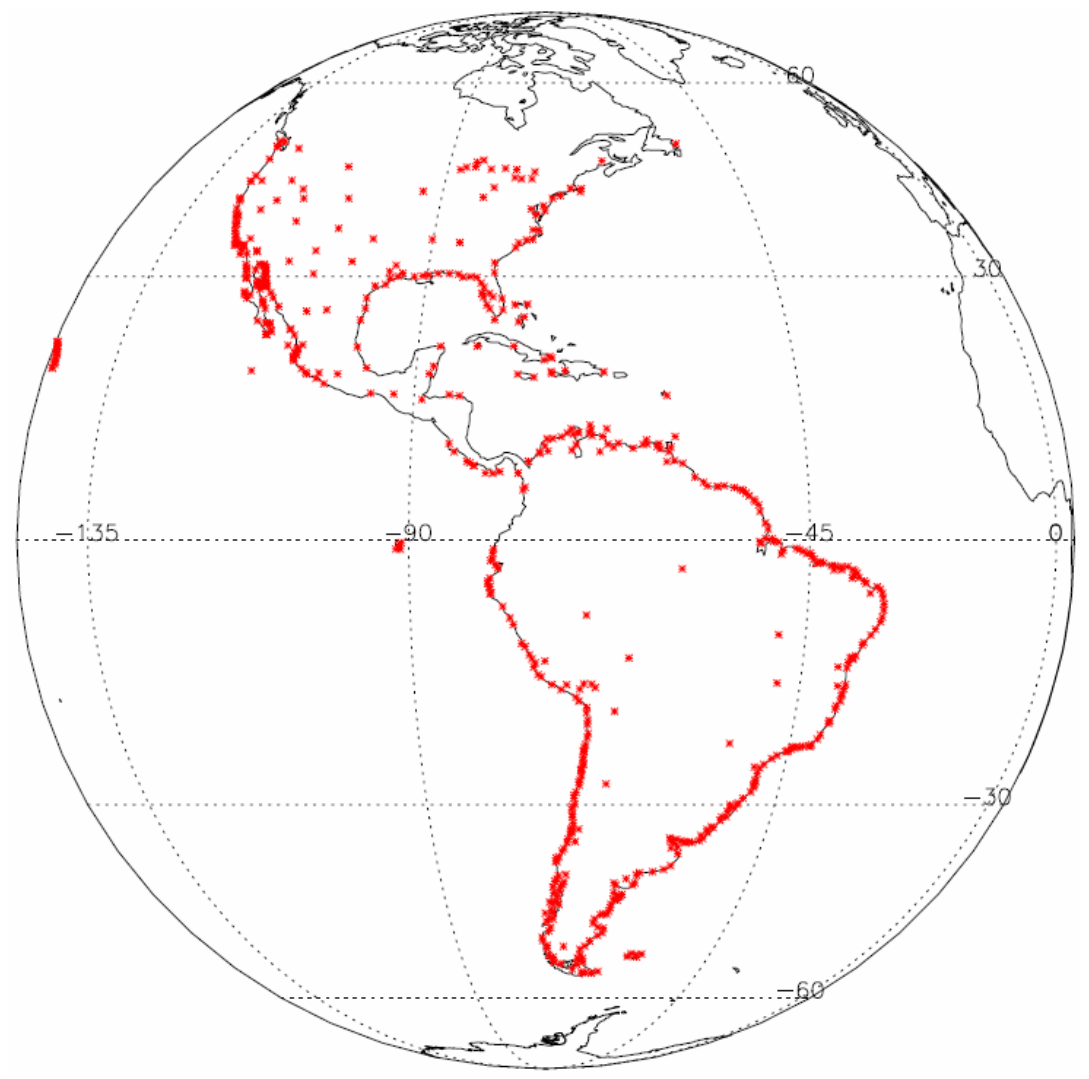

Figure 2. Locations of 630 Landsat chips. The chips cover North/South America and islands in west Pacific.

The Landsat chips are either cloud free or contain a small amount of cloud coverage, usually less than 5\%. Spectral response difference is a major error source when registering the images from different spectral channels. For example, the land/water boundary is the primary feature for matching $\mathrm{ABI}$ and Landsat subsets. The locations of the land/water boundary could be at different locations in different channels due to spectral response difference and ground feather characteristics 
e.g. steep or mild slope on the shoreline. Therefore, it is necessary to use a multiple-spectral chip library when assessing ABI NAV accuracy. Table 1 shows a comparison of the spectral channels of ABI and Landsat 8 side by side. For visible and near infrared (VNIR) channels, ABI and Landsat 8 channels show good spectral overlap. For mid-wave infrared (MWIR) and long-wave infrared (LWIR) channels, there is no close correspondence between ABI and Landsat 8 channels. We determined the match channels based on the spectral response characteristics and also made necessary adjustment after carefully examining ABI and Landsat subsets. Originally, we utilized channel 7 of Landsat 8 (short-wave infrared channel) to assess channel 7 of ABI (MWIR) [7]. After examining operational ABI data, we found that channel 10 of Landsat 8 (LWIR) is a better choice to assess ABI channel 7 considering night time emissions. Such adjustments occurred throughout the IPATS development and testing process.

Table. 1 ABI channels and the corresponding Landsat channels utilized for NAV measurements. The ABI atmospheric channels are excluded from the NAV measurements (and not included in this table) because they cannot see the ground and so cannot be compared with static Landsat chips.

\begin{tabular}{|c|c|c|c|c|c|}
\hline $\begin{array}{c}\text { GOES-16 } \\
\text { ABI Channel }\end{array}$ & $\begin{array}{c}\text { GOES-16 ABI } \\
\text { Wavelength (um) }\end{array}$ & $\begin{array}{c}\text { GOES-16 ABI } \\
\text { Spatial Resolution } \\
\text { (urad) }\end{array}$ & $\begin{array}{c}\text { Landsat } 8 \\
\text { Band }\end{array}$ & $\begin{array}{c}\text { Landsat } 8 \\
\text { Wavelength (um) }\end{array}$ & $\begin{array}{l}\text { Landsat } 8 \text { Spatial } \\
\text { Resolution (m) }\end{array}$ \\
\hline 1 & $0.45-0.49$ & 28 (1 $\mathrm{km}$ at nadir $)$ & 2 & $0.45-0.51$ & 30 \\
\hline 2 & $0.59-0.69$ & $14(0.5 \mathrm{~km}$ at nadir $)$ & 4 & $0.64-0.67$ & 30 \\
\hline 3 & $0.846-0.885$ & 28 (1 km at nadir) & 5 & $0.85-0.88$ & 30 \\
\hline 5 & $1.58-1.64$ & $28(1 \mathrm{~km}$ at nadir $)$ & 6 & $1.57-1.65$ & 30 \\
\hline 6 & $2.225-2.275$ & $56(2 \mathrm{~km}$ at nadir $)$ & 7 & $2.11-2.29$ & 30 \\
\hline 7 & $3.80-4.00$ & $56(2 \mathrm{~km}$ at nadir $)$ & \multirow{4}{*}{10} & \multirow{4}{*}{$10.60-11.19$} & \multirow{4}{*}{100} \\
\hline 11 & $8.3-8.7$ & 56 (2 $\mathrm{km}$ at nadir $)$ & & & \\
\hline 13 & $10.1-10.6$ & $56(2 \mathrm{~km}$ at nadir $)$ & & & \\
\hline 14 & $10.8-11.6$ & 56 (2 km at nadir) & & & \\
\hline 15 & $11.8-12.8$ & $56(2 \mathrm{~km}$ at nadir $)$ & \multirow{2}{*}{11} & \multirow{2}{*}{$11.50-12.51$} & \multirow{2}{*}{100} \\
\hline 16 & $13.0-13.6$ & $56(2 \mathrm{~km}$ at nadir $)$ & & & \\
\hline
\end{tabular}

\subsection{Chip registration}

In the IPATS NAV registration process, a subset of the ABI image is shifted around inside the Landsat chip and a correlation coefficient is retrieved for each shift position. The position of the highest coefficient, which indicates the best match between the ABI subset and the Landsat chip, marks the "true" location of the ABI image. The NAV error is defined as:

$$
E_{N A V}=L_{\text {nominal }}-L_{\text {true }} \quad \text { Eq. } 1
$$

Where $E_{N A V}$ is the NAV error of the subset of the ABI image. $L_{\text {nominal }}$ is the nominal ABI subset location assigned by the satellite data production systems. $L_{\text {true }}$ is the true ABI subset location assessed using the Landsat chip.

There are three image registration algorithms as mentioned in Sec. 2.1. In the operational assessments, Pearson Cross Correlation is applied because it requires the least computation time of the three algorithms while maintaining comparable accuracy. The Pearson Correlation Coefficient is calculated as:

$$
\gamma(\boldsymbol{u}, \boldsymbol{v})=\frac{\sum_{x, y}\left[f(x, y)-\overline{f_{u, v}}\right][t(x-u, y-v)-\bar{t}]}{\sqrt{\sum_{x, y}\left[f(x, y)-\overline{f_{u, v}}\right]^{2} \sum_{x, y}[t(x-u, y-v)-\bar{t}]^{2}}}
$$

Eq. 2

Where $(u, v)$ indicate the $(x, y)$ direction integer shift of the subset of GOES-16 image, $\gamma(u, v)$ is the Pearson correlation coefficient at the shifted location, $f(x, y)$ is the Landsat image value at pixel location $x$ and $y$, and $t(x-u, y-v)$ is the GOES-16 image value at pixel location $x-u$ and $y-v, \overline{f_{u, v}}$ is the mean of Landsat pixel values in the region overlapping with the GOES-16 subset, and $\bar{t}$ is the mean pixel value of the subset of GOES-16.

\subsection{Screening of the raw IPATS results}


The accuracy of the IPATS measurements depends the characteristics of the image pair. In addition to the differences between the ABI and Landsat sensor, other factors such as cloud coverage, seasonality, and image observation time, lead to additional registration errors between Landsat chips and the corresponding GOES-16 subsets. The measured NAV errors due to these factors could be as large as several GOES-16 pixels and so it is necessary to filter out these poor quality measurements to obtain a better estimate of the true NAV errors.

\subsubsection{Measurement uncertainty}

De Luccia et al. [7] introduced a parameter, measurement uncertainty $(M U)$ that quantitatively describes the quality of a measurement. Here it is revised from the original formula to improve the sensitivity to the measurement quality:

$$
M U=\frac{1}{s} \frac{1}{a} \sqrt{1-C C_{p k}^{2}} \frac{D}{N M} \frac{1}{2}\left(\frac{1}{c_{1}}+\frac{1}{c_{2}}\right)
$$

$a$ : peak sharpness, which is measured by the second derivative of Pearson correlation coefficient surface at the peak in each direction;

$C C_{p k}$ : Pearson correlation coefficient at the peak location;

$N$ and $M$ : the image dimensions;

$D$ : the normalized contrast difference between two images at overlap region;

$c 1$ and $c 2$ : normalized contrast of two images;

$S$ : the scale factor used to resample GOES-16 subsets to fine resolution to match Landsat chips when registering the image pair.

The peak sharpness is calculated in both the East-West (EW) and North-South (NS) direction. Therefore, $M U$ is also evaluated independently in the EW and NS direction. Figure 3 shows the relationship between the raw IPATS measurements and 1/MU in the EW direction. The plot is from GOES-16 channel 1 data acquired on April 11, 2018. The variation of raw measurements decreases quickly with increasing $1 / M U$ values, which indicates $M U$ represents the measurement quality very well. Currently, the $M U$ threshold is set to $0.357(1 / \mathrm{MU}=2.8)$. The measurements with an $M U$ value in the EW direction that are larger than the threshold are removed. A similar screening process is also applied on NS direction. 14028 of 24462 NAV measurements are removed by MU filters in the EW and NS directions.

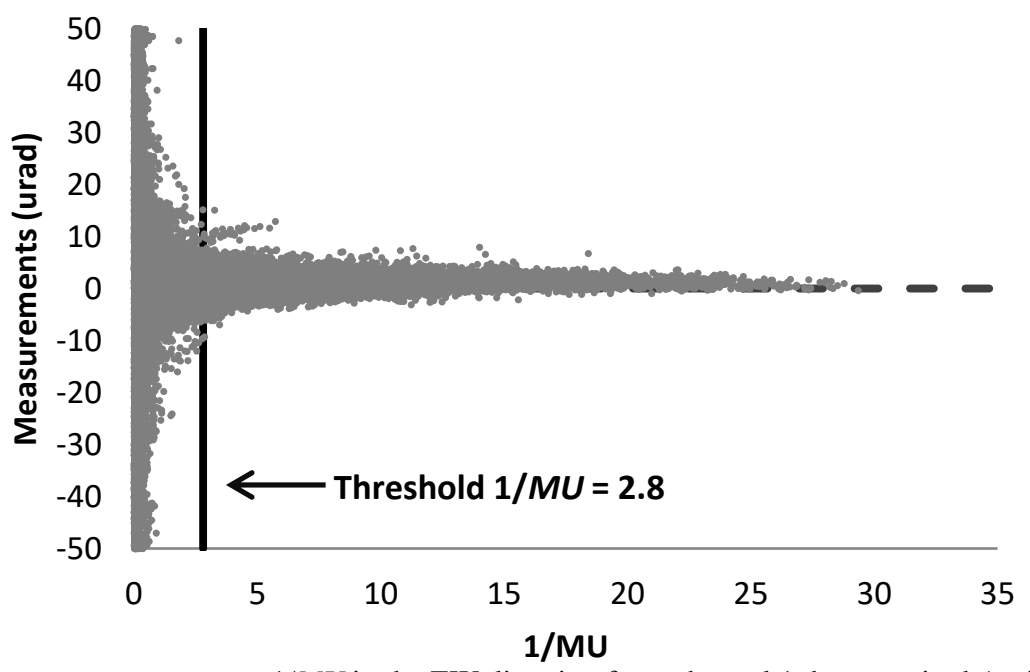

Figure 3. The raw IPATS measurements versus $1 / M U$ in the EW direction from channel 1 data acquired April 112018. The variation of raw measurements drops significantly with increasing $1 / M U$ value. 


\subsubsection{Statistics-based filters}

Most of the poor-quality NAV measurements are removed by the MU filter. Another post-processing filter, that uses the Median Absolute Deviation from the median (MAD), is applied to clean up remaining significant outliers. First, the MAD is calculated from all NAV measurements that passed the MU filter in a 24 hour period. A NAV measurement is then removed by the MAD filter when the absolute deviation of this measurement from the median of NAV measurements in 24 hours is larger than nine times the MAD value.

Occasionally, the MAD filter masks short-term abnormal situations by removing legitimate large measurements. For example, in a 24 hour period, if only a few scenes have significant (and real) NAV error, most of the NAV measurements in these scenes are removed by the MAD filter because of the out-of-family large NAV error readings. To capture a transient NAV error swing, a scene is marked as a "real significant error" scene if more than $50 \%$ measurements from a scene are removed by the MAD filter. The measurements from this scene are kept in the final report. The outliers of such a scene are determined and removed if the deviation is greater than three times the standard deviation of all the measurements in this scene. This process is named Short Term AbNormal Detection (STAND).

\section{RESULTS AND DISCUSSIONS}

\subsection{Long-term NAV tendency}

Long term ABI NAV results (Figure 4) capture the on-orbit calibration process of the GOES-16 ABI navigation system. After post-launch testing at the beginning of $2017, \mathrm{ABI}$ data quality reached provisional maturity on July $1^{\text {st }} 2017$. The GOES-16 satellite was moved from the GOES Central to the East position in early December 2017. There is no data available during this 12-day orbit drift maneuver.

Figures 4 and 5 are the time series plots of the daily mean and standard deviation of the GOES-16 ABI NAV FD errors from January 27, 2017 to May 31, 2018. Channels 2, 7, and 13 represent VNIR, MWIR, and LWIR focal plane modules, respectively. In general, NAV error in the NS direction is less than in the EW direction and is more stable over time.

From January 27 to April 27 2017, the NAV error in the EW direction of all channels and NS direction for channels 7 and 13 are significant. It should be noted that the gaps in channels 7 and 13 in January and April 2017 are due to the high EW NAV error, about - 80 urad, which are out of the plot range. The EW NAV error of channel 13 decreased from about 19 urad to about 4 urad as a result of navigation parameters updates on June 16 2017. And so, the GOES-16 navigation accuracy has been very good since it was released to the public as provisional on July 1, 2017.

TABLE 2. Mean NAV FD performance before and after the November 212017 and April 272018 updates. The before and after statistics are for the 24 hour periods ending at November 18 18:00 UTC and November 26 18:00 UTC, respectively for November 2017 update. For April 2018 update, the before and after statistics are for the 24 hour periods ending at April 24 18:00 UTC and May 01 18:00 UTC.

\begin{tabular}{|c|c|c|c|c|c|c|c|c|}
\hline \multirow{3}{*}{ Channel } & \multicolumn{4}{|c|}{ EW Misregistrations (urad) } & \multicolumn{4}{|c|}{ NS Misregistrations (urad) } \\
\hline & \multicolumn{2}{|c|}{ Nov Update } & \multicolumn{2}{|c|}{ April Update } & \multicolumn{2}{|c|}{ Nov Update } & \multicolumn{2}{|c|}{ April Update } \\
\hline & Before & After & Before & After & Before & After & Before & After \\
\hline 1 & 7.5 & 0.9 & 1.5 & -1.3 & 2.5 & 0.8 & 0.4 & 0.6 \\
\hline 2 & 7.4 & 0.3 & 1.4 & -1.7 & 2.8 & 0.5 & 0.2 & 0.2 \\
\hline 3 & 8.9 & 0.6 & 1.2 & -1.9 & 2.8 & 0.7 & 0.7 & 0.9 \\
\hline 5 & 7.7 & 0.6 & 1.5 & -1.6 & 2.1 & 0.5 & 0.6 & 0.4 \\
\hline 6 & 6.5 & 0.2 & 1.8 & -0.9 & 2.7 & 0.7 & 0.4 & -0.4 \\
\hline 7 & 5.8 & 1.1 & 2.4 & 0.3 & 2.5 & 0.1 & -0.6 & -0.3 \\
\hline 11 & 4.9 & 1.0 & 2.0 & 0.3 & 2.7 & -0.1 & -0.2 & -0.1 \\
\hline 12 & 4.8 & 1.0 & 2.0 & 0.3 & 0.7 & -0.7 & -0.7 & -0.4 \\
\hline 13 & 6.1 & 1.3 & 1.7 & -0.2 & 2.1 & -0.1 & -0.7 & -0.3 \\
\hline 14 & 5.6 & 1.0 & 1.5 & -0.1 & 1.5 & -0.4 & -0.9 & -0.4 \\
\hline 15 & 5.6 & 0.9 & 1.8 & 0.3 & 1.1 & -0.5 & -1.1 & -0.4 \\
\hline 16 & 5.9 & 1.0 & 1.8 & 0.3 & 1.8 & -0.3 & -1.0 & -0.1 \\
\hline
\end{tabular}



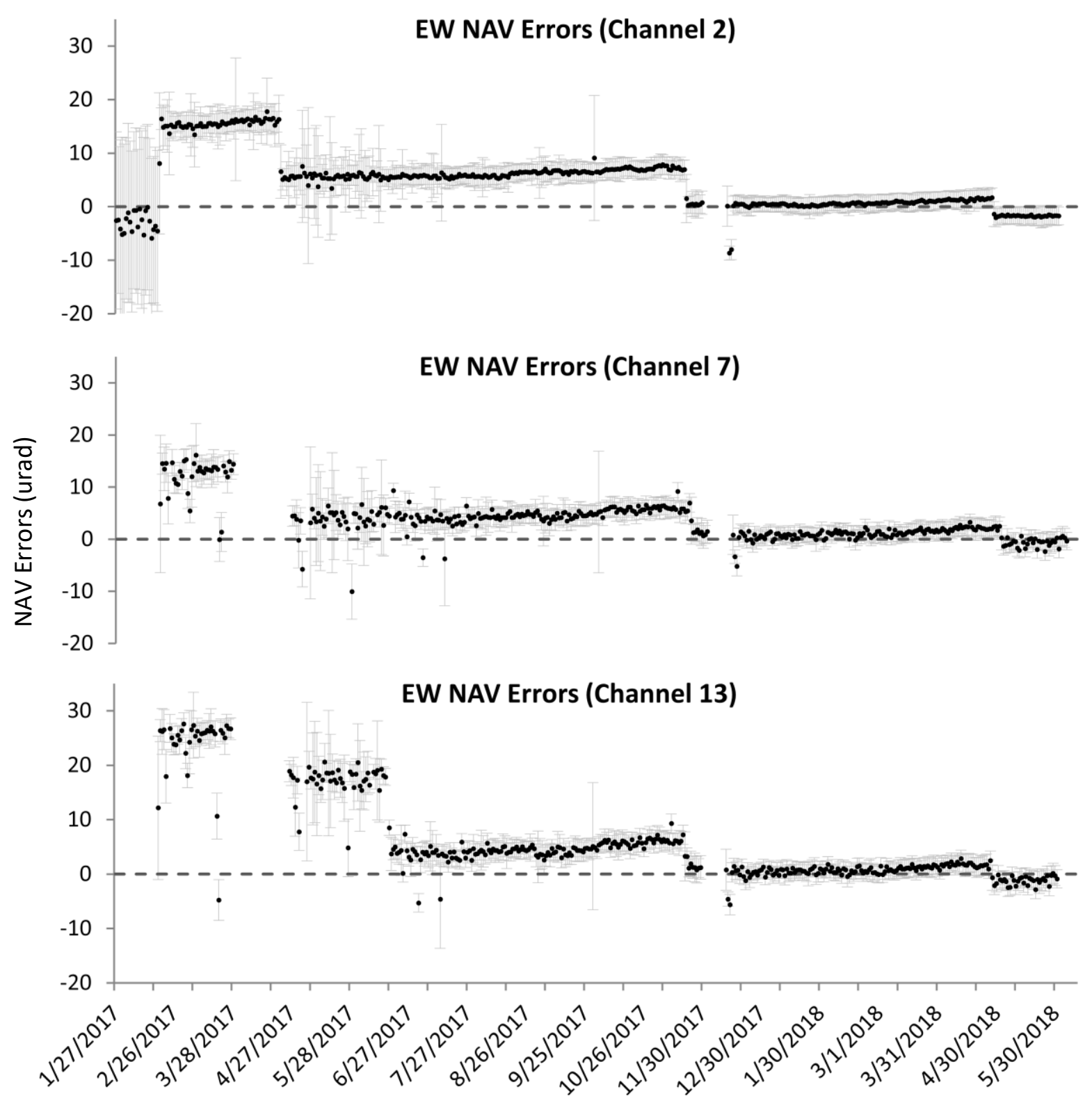

Figure 4. The daily mean and standard deviation of the GOES-16 ABI EW NAV errors for channel2 2, 7 and 13, representing VNIR, MWIR, and LWIR channels respectively.

The next important update was on November 21, 2017. After this update, the EW and NS NAV errors of all channels show a significant improvement (Table 2). The EW NAV errors dropped from about 5 urad to about 1 urad, about $3 \%$ of the pixel size, across all channels. The NS NAV errors dropped from about 2 urad to less than 1 urad in all channels as well. The latest update happened on April 27 2018. The EW NAV errors of MWIR and LWIR decreased from about 1.8 urad to less than 0.5 urad. However, The EW NAV errors for VNIR channels remains unchanged at about 1.5 urad, but flipped from eastward to westward. There is no change in the NS NAV errors after the April 2018 update.

There is a gradual increase in the EW NAV error between updates (Figure 4). The EW NAV error increased about 1 urad in all channels from June 2017 to Nov 2017 and from Nov 2017 to Apr 2018. This is due to the glitch in spacecraft orbit data processing, where a zonal tide term was missing. The missing term drifts slowly over time and led to the EW error 
gradually increasing as observed. The April 2018 updates included a ground system upgrade to accommodate the missing term by calculation and input of zonal tide approximation parameters. This should remove the slowly drifting error term in the spacecraft orbit data and fix the EW drift. This improvement will be verified when enough data are available.
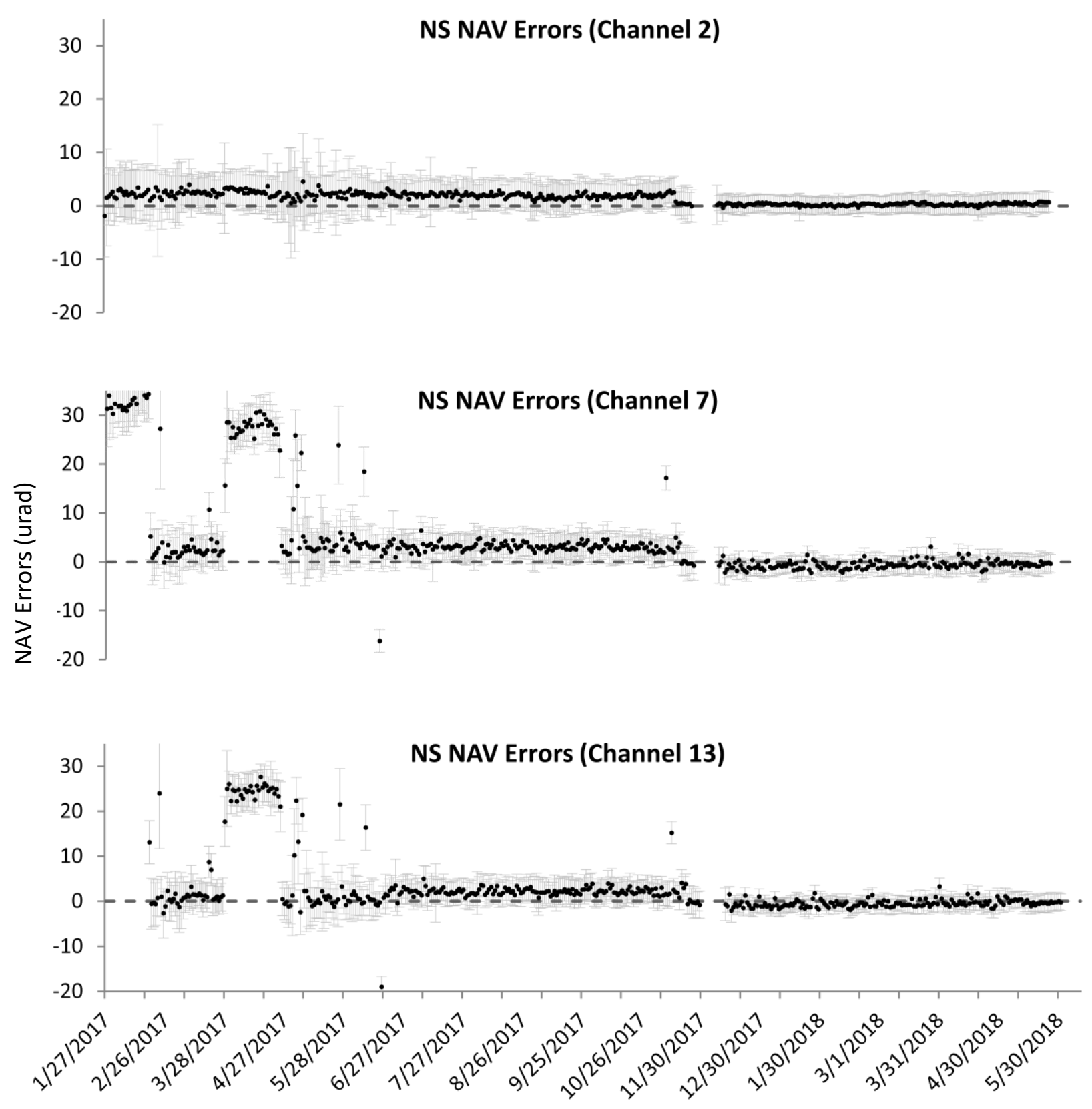

Figure 5. The daily mean and standard deviation of the GOES-16 ABI NS errors for channels 2, 7 and 13, representing VNIR, MWIR, and LWIR channels respectively. 


\subsection{Near real-time abnormality detection}

NAV measurements are not only good for assessing the overall image navigation system performance with daily and scenelevel statistics but also useful for detecting sudden sub-scene variations. During the eclipse season, fast thermal deformations in the sensor around penumbral times lead to abnormal, large image navigation error for a short time period. Figure 6 shows average swath NAV errors for channel 13 FD images from 04:15 UTC to 04:45 UTC on March 13, 2018. On that day, GOES-16 entered penumbra at 04:36:30 UTC and then exited penumbra and entered umbra at 04:38:47 UTC. The penumbral period is shorter than the acquisition time of a FD image (about 10 minutes). The FD image is composed of 22 swaths that are consecutive in time order with varying time intervals, with individual swath scanning from west to east and consecutive swaths located from north to south [6]. Therefore, the image distortion due to the penumbral effect only impacted the southern part of the FD image acquired around 04:30UTC. The distortion is about 40 urad in the EW direction. The image NAV accuracy of the northern part of the image remained at normal level, the same as FD images acquired before (04:15 UTC) and after (04:45 UTC).

(a)

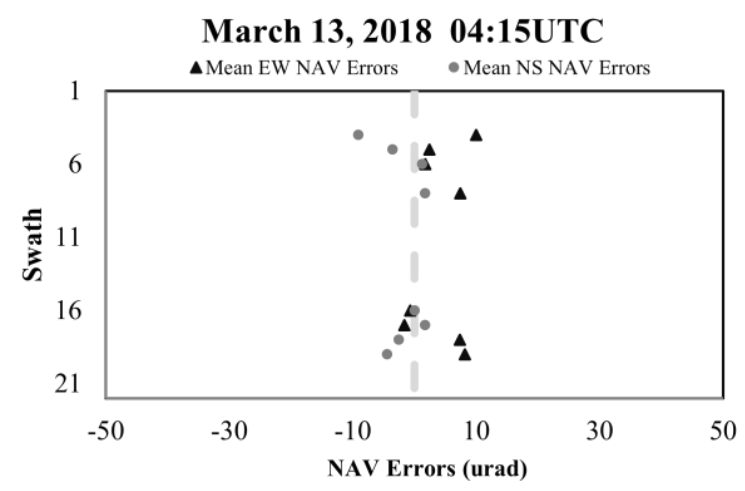

(c)

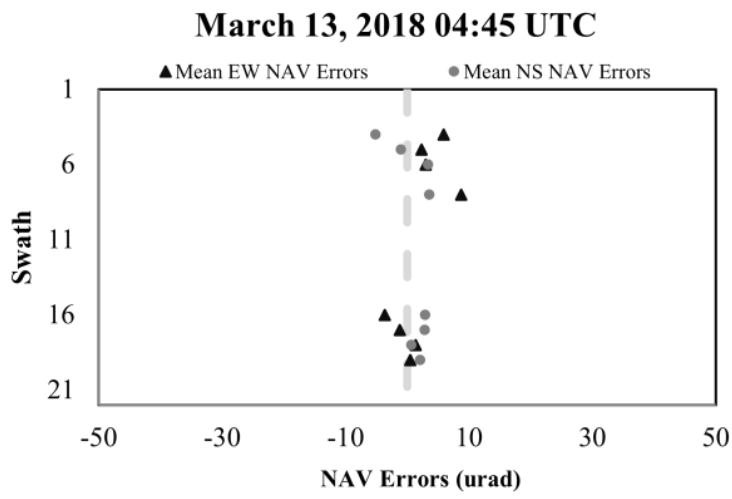

(b)

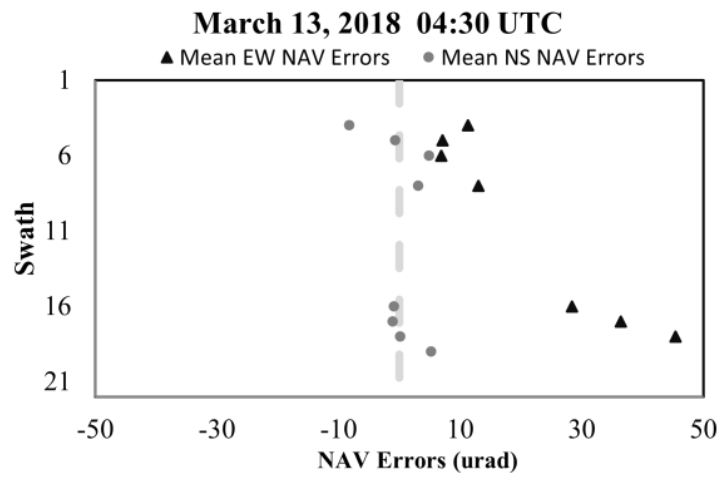

Figure 6. GOES-16 ABI Channel 13 mean swath NAV errors for the three scenes acquired from 04:15 UTC to 04:45 UTC on March $13,2018$.

\subsection{NAV measurements for in-depth analysis}

In addition to directly assessing NAV errors, the estimated NAV errors are also useful to evaluate and understand satellite attitude anomalies, focal plan misalignment, scan encoder misalignment, etc. Figure 7 show the relationship between the NAV FD measurement readings and latitude/longitude based on 30 days of NAV results in September 2017, April 2018 and May 2018 respectively. 
In September 2017 (Panel a and b in Figure 7), the scale errors (NS vs. latitude and EW vs. longitude) are pretty strong ( $\mathrm{R}^{2}$ of 0.94 and 0.65 , respectively) and in the same direction. The yaw errors (NS vs. longitude and EW vs. latitude) are in same directions and strong $\left(\mathrm{R}^{2}\right.$ of 0.66 and 0.79 , respectively). It should be noted that the yaw errors measured here is not the spacecraft attitude, but the combination of the spacecraft attitude and ABI to spacecraft misalignment. The difference in magnitude of the slopes -0.04 vs -0.01 , may indicate an uncompensated non-orthogonality in the scan axis.

(a)

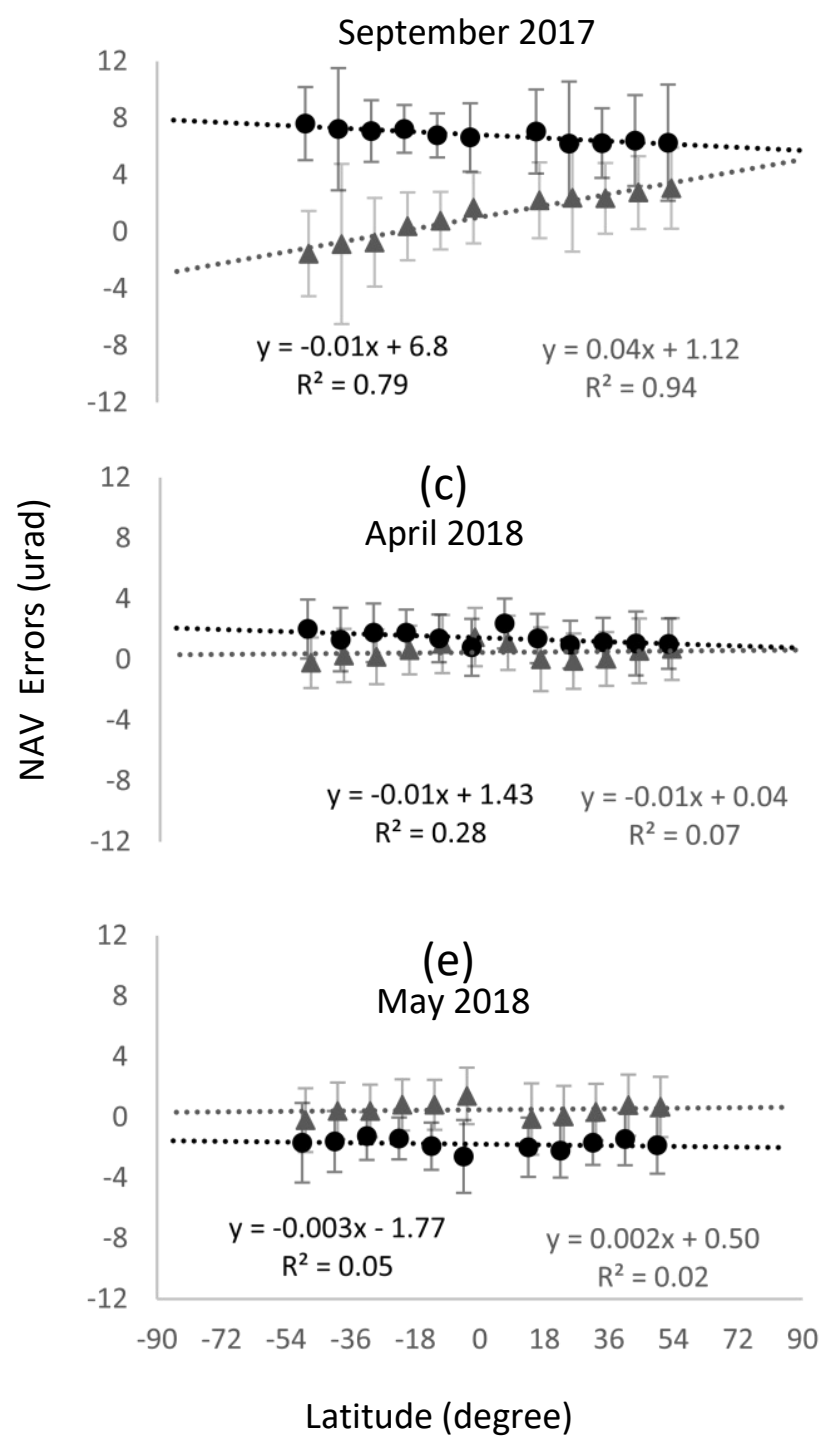

(b)

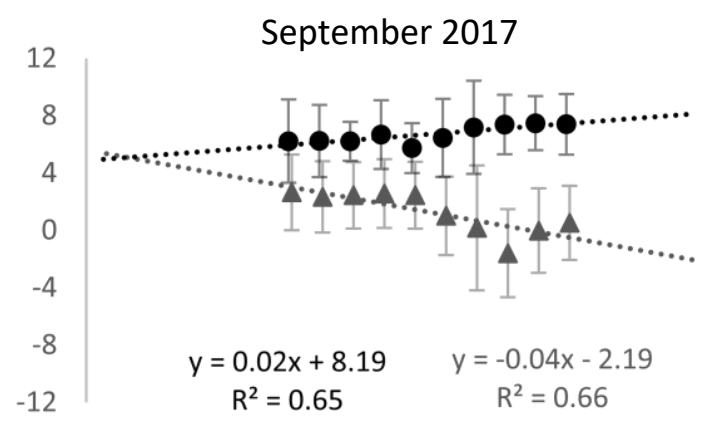

(d)

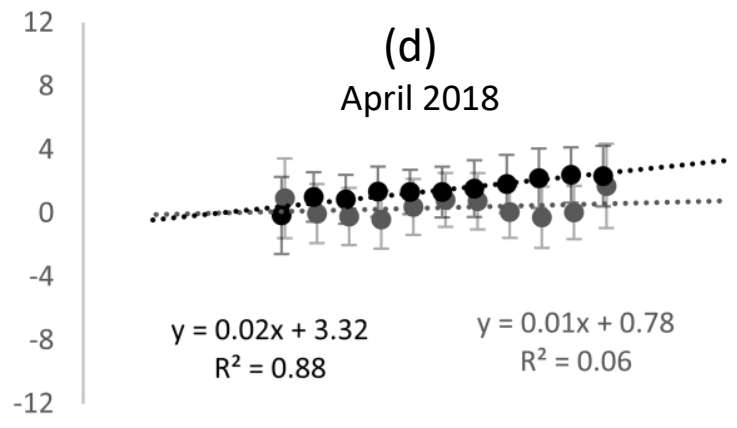

(f)

May 2018

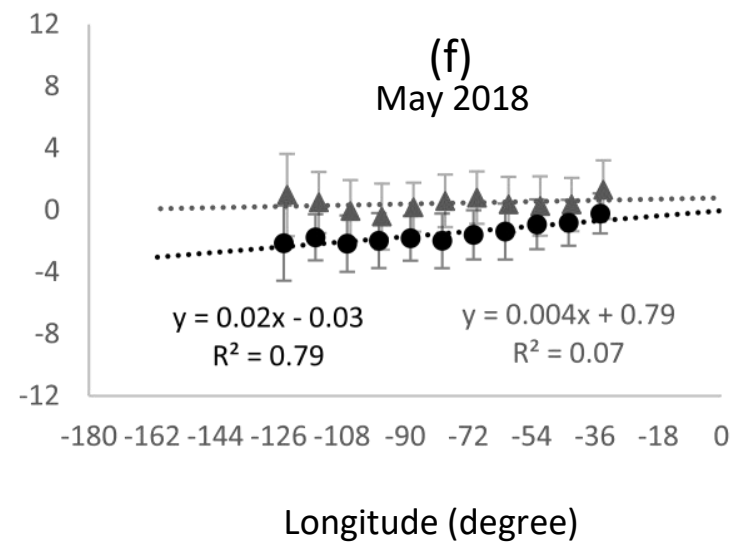

Legend $\mathrm{EW}$

Figure 7. GOES-16 monthly mean and standard deviation of NAV errors of FD Channel 2 against latitude and longitude. Panels a and b are from September 2017 data. Panels c and d are from April 2018. Panels e and f are from May 2018. It should be noted that only statistic bins with more than 500 chip matchups in 30 days are included. 
In April 2018, after the November 2017 updates, the scale error is negligible in the NS direction ( $\mathrm{R}^{2}$ of 0.07 for NS vs. latitude) but became stronger in the EW direction ( $\mathrm{R}^{2}$ of 0.88 for $\mathrm{EW}$ vs. longitude). The yaw errors are not significant ( $\mathrm{R}^{2}$ of 0.28 and 0.06 for EW vs. latitude and NS vs. longitude respectively).

In May 2018, after the April 2018 updates, the scale error stays negligible in the NS direction ( $\mathrm{R}^{2}$ of 0.02 for NS vs. latitude) and stays significant in the EW direction ( $\mathrm{R}^{2} 0.79$ for $\mathrm{EW}$ vs. longitude). The effect of yaw attitude are minimized ( $\mathrm{R}^{2}$ of 0.02 and 0.07 for EW vs. latitude and NS vs. longitude respectively).

Future trending will continue to identify any systematic errors that could be corrected and verify the improvement of each update.

\section{CONCLUSIONS}

IPATS has been designed and implemented to generate many ABI INR metrics and has been used to understand and help with the tuning of the GOES-16 ABI navigation system to achieve excellent image navigation and registration accuracy requirements. This study shows that GOES-16 ABI NAV has improved from launch to the end of May 2018. Currently, NAV errors are about 1.5 urad or less for all assessed channels. NAV assessments have demonstrated improved image navigation results and will continue to provide feedback for tuning the navigation algorithms and parameters in future updates.

\section{ACKNOWLEDGEMENT}

The IPATS activity is supported by the NASA/NOAA GOES-R Series Project. The authors acknowledge contributions by the many members of the GOES-R Series Flight Project (including the spacecraft and instrument designers and builders), the GOES-R Series Ground Project and the NOAA GOES-R Series Calibration Working Group, whose efforts make these results possible. Particularly thanks go to Dr. Frank J. De Luccia and Dr. Evan Hass, the leads of the IPATS team.

\section{REFERENCE}

[1] Miller, S. D., Schmidt, C. C., Schmit, T. J. and Hillger, D. W. “ A case for natural colour imagery from geostationary satellites, and an approximation for the GOES-R ABI", International Journal of Remote Sensing, 33:13, 3999-4028, DOI: 10.1080/01431161.2011.637529 (2012).

[2] Chapel, J., Stancliffe, D., Bevacqua, T., Winkler, S., Clapp, B., Rood, T., Gaylor, D., Freesland, D. and Krimchansky, A. "Guidance, Navigation, and Control Performance for the GOES-R Spacecraft", CEAS Space Journal, DOI 10.1007/s12567-015-0077-1 (2015).

[3] Schmit, T. J., Griffith, P., Gunshor, M. M., Daniels, J. M., Goodman, S. J. and Lebair, W. J. "A closer look at the ABI on the goes-r series. Bulletin of the American Meteorological Society", 98(4), 681-698. DOI 10.1175/BAMS-D-1500230.1 (2017).

[4] Tan, B., Woodcock, C.E, Hu, J., Zhang, P., Ozdogan, M., Huang, D., Yang, W., Knyazikhin, Yu., and Myneni, R. B. "The impact of gridding artifacts on the local spatial properties of MODIS data: Implications for validation, compositing, and band-to-band registration across resolutions", Remote Sensing of Environment, vol.105, 98-114 (2006).

[5] Roy, D. P. "The impact of misregistration upon composited wide field of view satellite data and implications for change detection", IEEE Transactions on Geoscience and Remote Sensing, Vol 38, 2017-2032 (2000).

[6] 'Product Definition and Users' Guide (PUG) Volume 3: Level 1B Products for Geostationary Operational Environmental Satellite R Series (GOES-R) Core Ground Segment, Revision D”, http://www.goesr.gov/users/docs/PUG-L1b-vol3.pdf (May 2015).

[7] De Luccia, F. J., Houchin, S., Porter, B. C., Graybill, J., Haas, E., Johnson, P. D., Isaacson, P. J., Reth, A. D. "Image navigation and registration performance assessment tool set for the GOES-R Advanced Baseline Imager and 
Geostationary Lightning Mapper”, Proc. SPIE 9881, Earth Observing Missions and Sensors: Development, Implementation, and Characterization IV, 988119 (2 May 2016); doi: 10.1117/12.2229059; https://doi.org/10.1117/12.2229059 (2016).

[8] "Geostationary Operational Environmental Satellite (GOES) GOES-R Series Advanced Baseline Imager Performance and Operational Requirements Document (PORD)", Baseline Version 2.37.1 (Oct 2016).

[9] Storey, J., Choate, M. and Lee, K. "Landsat 8 Operational Land Imager On-Orbit Geometric Calibration and Performance", Remote Sensing 6(11), 11127-11152; DOI:10.3390/rs61111127 (2014). 